\title{
The Masses of $P_{c}^{*}(4380)$ and $P_{c}^{*}(4450)$ as Di-Hadronic States
}

\author{
Rismita Ghosh', Aparajita Bhattacharya ${ }^{1}$, Ballari Chalrabarti² \\ ${ }^{1}$ Department of Physics, Jadavpur University, Kolkata, India \\ ${ }^{2}$ Department of Physics, Jogamaya Devi College, Kolkata, India \\ Email: rismita.ghosh@gmail.com, pampa@phys.jdvu.ac.in, ballari_chakrabarti@yahoo.co.in
}

Received 7 October 2015; accepted 17 November 2015; published 20 November 2015

Copyright (C) 2015 by authors and Scientific Research Publishing Inc.

This work is licensed under the Creative Commons Attribution International License (CC BY).

http://creativecommons.org/licenses/by/4.0/

\section{(c) (i) Open Access}

\section{Abstract}

The masses of the recently reported by LHCb two pentaquark charmonium states $P_{c}^{*}(4380)$ and $P_{c}^{*}(4450)$ which are suggested to possess pentaquark configuration (uudc $\bar{c}$ ) have been estimated considering a di-hadronic state consisting of a meson $c \bar{c}$ and a baryon (uud). The binding energies of the states have been estimated with a van der Walls type of molecular interaction between the hadrons. A spin interaction has also been considered. Masses of these two states are well reproduced with the aforesaid molecular interaction which indicates that the multiquarks $P_{c}^{*}(4380)$ and $P_{c}^{*}(4450)$ can be well described as meson-baryon bound states.

\section{Keywords}

\section{Pentaquark, Di-Hadronic Molecule, Molecular Interaction}

\section{Introduction}

The existence of pentaquark charmonium states with the decay of $\Lambda_{b}^{0}\left(\Lambda_{b}^{0} \rightarrow J / \psi K^{-} p\right)$ has been reported by LHCb [1] recently. The intermediate states have been identified as $P_{c}^{*}(4380)$ and $P_{c}^{*}(4450)$. The states are identified as sum of two up quarks, one down quark, one charm quark and one anti-charm quark with spin $\frac{3}{2}$ and $\frac{5}{2}$ respectively. The identification of these pentaquark states is exciting and will give new impetus to the study of the properties and dynamics of multiquark states [2]. The exotics remain less known and less unders- 
tood compared to the properties of mesons ( $\mathrm{q} \overline{\mathrm{q}}$ ) and baryons (qqq) which are well studied both in theory and experiment. The properties and dynamics of exotic states like tetraquark, pentaquark, hexaquark states are yet to be studied and it is well understood that they cannot be described in the framework of conventional quark model. A number of models like the quark model [3] [4], bag model [5] [6], and non-relativistic potential models [7] [8] have been used to study the multiquark systems. The description of the multiquark states as di-hadronic states considering them as a bound state of a meson and a baryon is one of the useful candidates for studying the properties of such systems.

\section{Method}

In the present work pentaquark states $P_{c}^{*}(4380)$ and $P_{c}^{*}(4450)$ are described as di-hadronic molecules consisting of a meson and a baryon assuming a van der Waals type of molecular interaction acting between the constituent hadrons [9] [10]. $\mathbf{P}_{c}^{*}$ (4380) state of spin $\frac{3}{2}$ is assumed to have configuration as proton-charmonium state as ( uudc $\overline{\mathrm{c}}$ ) whereas $P_{c}^{*}$ (4450) state of spin $\frac{5}{2}$ has been described as $\Delta-\mathrm{J} / \psi$ state. A spin interaction has also been considered. Masses of $\mathrm{P}_{\mathrm{c}}^{*}$ (4380) and $\mathrm{P}_{\mathrm{c}}^{*}$ (4450) as di-hadronic molecule have been estimated using the mass formula.

Assuming the pentaquark states as meson-baryon system the mass formula for the low-lying di-hadronic molecule runs as:

$$
M_{\text {Total }}=M_{1}+M_{2}+E_{B E}+E_{S D}
$$

where $M_{1}, M_{2}$ represent the masses of the constituent hadrons respectively, $E_{B E}$ represents the binding energy of the di-hadronic system and $E_{S D}$ represents the spin-dependent term.

The binding energy can be expressed as:

$$
E_{B E}=\langle\Psi(r)|V(r)| \Psi(r)\rangle
$$

where $r$ is the radius parameter of the di-hadronic molecule and $V(r)$ is the di-hadronic molecular potential which is expressed as [9] [10]:

$$
V(r)=\frac{-k_{\mathrm{mol}}}{r} e^{-C^{2} r^{2} / 2}
$$

where $k_{\mathrm{mol}}$ [9] [10] is the residual strength of the strong interaction molecular coupling and $C$ is the effective colour screening of the confined gluons. It may be mentioned that the residual interaction of the confined gluon is considered similar to van der Waals interaction and is assumed to be due to asymptotic expression $\left(r_{12} \rightarrow \infty\right)$ of the residual confined one-gluon exchange interaction with strength $k_{\text {mol }}$ [9] [10].

$\Psi(r)$ is the wave function of the di-hadronic state. To estimate $E_{B E}$ we have used the wave functions for the ground state of the hadronic molecule from statistical model which runs as: [11] [12]

$$
\begin{gathered}
|\Psi(r)|^{2}=\frac{315}{64 \pi r_{12}^{\frac{9}{2}}}\left(r_{12}-r\right)^{\frac{3}{2}} \theta\left(r_{12}-r\right) \\
|\Psi(r)|^{2}=\frac{8}{\pi^{2} r_{12}^{6}}\left(r_{12}^{2}-r^{2}\right)^{\frac{3}{2}} \theta\left(r_{12}-r\right)
\end{gathered}
$$

corresponding to the linear type of background potential and harmonic type of background potential respectively [11] [12]. $r_{12}$ is the radius of the hadronic molecule and $\theta\left(r_{12}-r\right)$ is usual step function. With $r_{12}=r_{1}+r_{2}$, where $r_{1}$ and $r_{2}$ represent the individual radii of the hadrons constituting the molecule respectively and using Equations (2), (3) and (4) we get $E_{B E}$ as:

$$
E_{B E}=\frac{2.25 k_{\mathrm{mol}}}{r_{12}} 2 F_{2}[(1,1.5),(2.25,2.75),-\beta]
$$




$$
E_{B E}=\frac{16 k_{\mathrm{mol}}}{\pi r_{12}^{5} C^{5}}\left[-6 C+2 r_{12}^{2} C^{3}+\frac{3 e^{-\beta}(2 \pi)^{\frac{1}{2}} \operatorname{Erfi}\left[\beta^{\frac{1}{2}}\right]}{r_{12}}\right]
$$

where $\beta=C^{2} r_{12}^{2} / 2, C=50 \mathrm{MeV}$ [13] and $k_{\mathrm{mol}}=0.59$ and 0.65 [14] corresponding to linear and harmonic type of background potentials respectively. The radius of the corresponding baryons have been estimated by adjusting the value of charge radii [15] [16] against the form factor of corresponding baryon [17] [18]. The radius of "p" and " $\Delta$ " are obtained as $7.59 \mathrm{GeV}^{-1}$ and $5.977 \mathrm{GeV}^{-1}$ respectively. The radius of $c \bar{c}$ has been used from [19] as $r(J / \psi)=2.005 \mathrm{GeV}^{-1}$.

The spin hyperfine interaction can be expressed as [20]:

$$
E_{S D}=\frac{8}{9} \frac{\alpha_{s}}{M_{1} M_{2}} S_{1} \cdot S_{2}|\Psi(0)|^{2}
$$

where $M_{1}$ and $M_{2}$ are the masses of the constituent hadrons in the di-hadronic molecule, $\alpha_{s}$ is the strong interaction constant, $S_{1}$ and $S_{2}$ are the spins of the hadrons involved, $|\Psi(0)|^{2}$ is the di-hadronic wave function at the origin. With $\alpha_{s}=0.59$ [21] the $E_{S D}$ has been estimated subsequently using the relation (8). The masses of $\mathrm{P}_{\mathrm{c}}^{*}$ (4380), $\mathrm{P}_{\mathrm{c}}^{*}$ (4450) have been estimated using the Equation (1) with mass of the respective meson $\left(M_{1}\right)$ and baryon $\left(\mathrm{M}_{2}\right)$ [15] [22] and displayed at the Table 1.

\section{Discussions}

In the present work we have estimated masses of particles $P_{c}^{*}(4380)\left(\frac{3}{2}\right)$ and $P_{c}^{*}$ (4450) $\left(\frac{5}{2}\right)$ considering them as di-hadronic (meson-baryon) molecules. The masses have been obtained as $4171 \mathrm{MeV}$ and 4492 $\mathrm{MeV}$ for $\mathrm{P}_{\mathrm{c}}^{*}$ (4380) and $4168 \mathrm{MeV}$ and $4191 \mathrm{MeV}$ for $\mathrm{P}_{\mathrm{c}}^{*}$ (4450) with the input of two wave functions from the statistical model. The results are found to be in good agreement with the experiment. We have observed that the mass of $\mathrm{P}_{\mathrm{c}}^{*}$ (4450) state is well reproduced where as for the $\mathrm{P}_{\mathrm{c}}^{*} \quad(4380)$ has somewhat smaller $(\sim 200 \mathrm{MeV})$ value which may be attributed to the uncertainty in the radius parameter used. It is interesting to note here that the pentaquarks $\mathrm{P}_{\mathrm{c}}^{*}\left(\frac{3}{2}\right), \mathrm{P}_{\mathrm{c}}^{*}\left(\frac{5}{2}\right)$ are well described in the di-hadronic molecules with a weak van der Waals type of interaction between them. It is also pertinent to point out that the statistical model wave function is also very successful in describing the hadrons. The pentaquark state is one of the leading candidates for the study of the multiquark state. The description of pentaquark as diquark-diquark-antiquark state has been done by a number of authors [3] [4]. It may be mentioned that recently some predictions of diquark model for hidden charm pentaquarks $\mathrm{P}_{\mathrm{c}}^{*}(4450)$ and $\mathrm{P}_{\mathrm{c}}^{*}(4380)$ have been proposed by Li et al. [23]. They have found that in the SU(3) limit, for U-spin related decay modes the ratio of the decay rates of Cabibbo suppressed to Cabibbo allowed decay channels is given by $\frac{\left|V_{c d}\right|^{2}}{\left|V_{c s}\right|^{2}}$. The present investigation shows that they are also described well in the framework of di-hadronic states.

\begin{tabular}{|c|c|c|c|c|c|c|c|c|}
\hline \multicolumn{6}{|c|}{ With $|\Psi(0)|^{2}$ from (4) } & \multicolumn{3}{|c|}{ With $|\Psi(0)|^{2}$ from (5) } \\
\hline & Particle & & State & $E_{B E}+E_{S D}(\mathrm{MeV})$ & $M(\mathrm{MeV})$ & $E_{B E}+E_{S D}(\mathrm{MeV})$ & $M(\mathrm{MeV})$ & $M_{\text {exp }}(\mathrm{MeV})[1]$ \\
\hline $\mathrm{P}_{\mathrm{c}}^{*}$ & (4380) (spin & $\left.\frac{3}{2}\right)$ & $\mathrm{p}-\mathrm{J} / \psi$ & 136 & 4171 & 133 & 4168 & $4380 \pm 8 \pm 29$ \\
\hline$P_{c}$ & (4450) (spin & $\left.\frac{5}{2}\right)$ & $\Delta-\mathrm{J} / \psi$ & 163 & 4492 & 162 & 4491 & $4449.8 \pm 1.7 \pm 2.5$ \\
\hline
\end{tabular}

Table 1. Binding energies and masses of pentaquark charmonium states as meson-baryon states. 


\section{Acknowledgements}

Authors are thankful to University Grants Commission, New Delhi, India for their financial supports.

\section{References}

[1] Aaij, R., et al. (LHCb Collaboration) (2015) Physical Review Letters, 115, Article ID: 072001(50).

[2] Gell-Mann, M. (1964) Physics Letters, 8, 214-215. http://dx.doi.org/10.1016/S0031-9163(64)92001-3

[3] Jaffe, R.L., et al. (2003) Physical Review Letters, 91, Article ID: 232003(1-4).

[4] Karlinar, M., et al. (2003) Physics Letters B, 575, 249-255. http://dx.doi.org/10.1016/j.physletb.2003.09.062

[5] Jaffe, R.L. (1977) Physical Review D, 15, 281-289. http://dx.doi.org/10.1103/PhysRevD.15.281

[6] Strottman, D. (1979) Physical Review D, 20, 748-767. http://dx.doi.org/10.1103/PhysRevD.20.748

[7] Barnes, T., et al. (1987) Physical Review Letters B, 183, 210. http://dx.doi.org/10.1016/0370-2693(87)90440-0

[8] Weinstein, J., et al. (1983) Physical Review D, 27, 588. http://dx.doi.org/10.1103/PhysRevD.27.588

[9] Vinodkumar, P.C., et al. (1992) Pramana-Journal of Physics, 39, 47-70.

[10] Vinodkumar, P.C., et al.(2003) DAE-BRNS Symposium on Nuclear Physics, 46B, 334.

[11] Bhattacharya, A., et al. (1998) European Physical Journal C, 2, 671-673.

[12] Bhattacharya, A., et al. (2005) Nuclear Physics (Proceedings Supplements), B142, 13. http://dx.doi.org/10.1016/j.nuclphysbps.2005.01.002

[13] Rai, A.K., et al. (2006) Indian Journal of Physics, 80, 387-392.

[14] Chakrabarti, B., et al. (2009) Physica Scripta, 79, Article ID: 025103. http://dx.doi.org/10.1088/0031-8949/79/02/025103

[15] Mohr, P.J., et al. (2011) The 2010 CODATA Recommended Values of the Fundamental Physical Constants. National Institute of Standards and Technology, Gaithersburg.

[16] Alexandrou, C., et al. (2007) PoSLAT2007, 149.

[17] Banerjee, S.N., et al. (1987) International Journal of Modern Physics A, 2, 1829. http://dx.doi.org/10.1142/S0217751X87000958

[18] Bhattacharya, A., et al. (1988) Canadian Journal of Physics, 66, 749-751. http://dx.doi.org/10.1139/p88-123

[19] Hong, C., et al. (2001) Chinese Physics Letters, 18, 1558. http://dx.doi.org/10.1088/0256-307X/18/12/305

[20] Rai, A.K., et al. (2007) Nuclear Physics A, 782, 406-409. http://dx.doi.org/10.1016/j.nuclphysa.2006.10.023

[21] Lucha, W., et al. (1991) Physics Report, 200, 168. http://dx.doi.org/10.1016/0370-1573(91)90001-3

[22] Beringer, J., et al. (Particle Data Group) (2012) Physical Review D, 86, Article ID: 010001. http://dx.doi.org/10.1103/PhysRevD.86.010001

[23] Li, G.N., et al. (2015) arXiv:1507.08252v2 [hep-ph]. 